\title{
Quantitative Limulus lysate assay for endotoxin and the effect of plasma
}

\author{
J C Hurley, F A Tosolini, W J Louis
}

\begin{abstract}
The effects of plasma and chromogenic substrate on the kinetics of the endotoxin-activated Limulus amoebocyte lysate (LAL) assay were determined. A linear correlation was observed between the rate of development of turbidity (optical density 405) with the LAL reagent and the concentration of endotoxin over a four log ten-fold range. Like chromogenic substrate, the addition of dilution and heat treated plasma to the reaction resulted in an increase in optical density proportional to the concentration of plasma present. The presence of the treated plasma also resulted in an accelerated increase in optical density with comparable results when testing plasma at different concentrations and, additionally, serum. This accelerated increase in optical density may not be recognised in assays that monitor the progress of the reaction at a single time point and may confound assays of plasma samples that use chromogenic substrate. Plasma obtained from endotoxin sensitive and resistant strains of mice showed similar effects.

The use of kinetic methodology means that a quantitative assay for endotoxin in plasma can be achieved, its variability comparable with that seen with semiquantitative serial dilution but with greater economy of the LAL reagent.
\end{abstract}

Following the pioneering work of Levin and Bang, ${ }^{1}$ the Limulus amoebocyte lysate (LAL) assay has gained increasing acceptance as a sensitive method for the detection of endotoxin (lipopolysaccharide). ${ }^{2}$ In the endotoxin-activated LAL reaction, the development of turbidity (optical density) with time describes a sigmoid curve with three phases-a lag phase, a period of rapid increase in optical density, and a terminal plateau phase. The absolute increase in optical density is a function of the concentration of LAL clottable protein, and the rate of increase in optical density is a function of the concentration of endotoxin. ${ }^{3}$

The LAL assay has been used to detect endotoxin in body fluids such as urine, cerebrospinal fluid, and urethral secretions. ${ }^{2}$ Use of the assay to detect endotoxin in blood, however, is hampered by a complex interaction between endotoxin, the LAL, and plasma, for which there are at least three mechanisms: endotoxin inactivation by temperature sensitive esterases in plasma and serum $^{45}$; an interaction between the LAL coagulogen and heat affected fibrinogen $;^{6}$ and an amplification of endotoxin activity through interaction with high density lipoproteins. ${ }^{7}$ Measurements of the progress of the LAL reaction at any single time point, although a convenient and commonly used method for endotoxin quantitation, also produce standard curves that are sigmoid in shape with respect to endotoxin concentration. ${ }^{89}$

Attempts by several groups of investigators to overcome these limitations have resulted in various modifications to the assay method. Three recent modifications to the $\mathrm{LAL}$ assay include the use of a chromogenic substrate as a substitute for the gelation reaction to give a more direct measure of LAL proenzyme activation, ${ }^{10}$ the dilution and heat inactivation of plasma to control the interfering factors in plasma, ${ }^{11}$ and the use of kinetic analysis as opposed to end point methods for quantitation of proenzyme activity. ${ }^{12}$ Considerable difficulties remain for quantitation of endotoxin in plasma, however. For example, Cohen and McConnell described an endotoxin assay in which chromogen conversion by activated LAL was monitored by kinetic techniques. ${ }^{13}$ Despite conventional heating and dilution to inactivate non-specific interfering factors in plasma, the variation in recovery of endotoxin added to samples of blood was more than $100 \%$. Moreover, this was not a strictly kinetic LAL assay as the period of kinetic monitoring did not correspond to the period of LAL activation. ${ }^{14}$

An important aspect not previously considered is that the presence of plasma may change the kinetics of the LAL reaction. This effect may not be recognised with single time point readings of turbidity, yet may have important consequences for quantitative $\mathrm{LAL}$ assays for endotoxin in plasma using end point methods. In this study we developed a quantitative microtitre plate assay for endotoxin using kinetic analysis. We compared two methods of kinetic analysis of the reaction of LAL with endotoxin and we investigated the effects of heat treated $10 \%$ plasma and of chromogenic substrate on the kinetics of the reaction.

\section{Methods}

PYROGEN FREE MATERIALS AND REAGENTS

All glassware was washed in distilled water and rendered free of endotoxin by heating at $220^{\circ} \mathrm{C}$ for five hours. 
Limulus amoebocyte lysate (LAL, Pyrogent, MA Bio Products Inc, Walkersville, USA) was reconstituted with $5.2 \mathrm{ml}$ of pyrogen free distilled water BP (DW, Water For Injection, David Bull, North Ryde, NSW, Australia), according to the manufacturer's instructions, and used the same day or stored at $4^{\circ} \mathrm{C}$ for use within seven days. All experiments were performed using LAL from the same lot (lot number 6LW 125). Chromogenic substrate (S2423, Kabi Vitrum, Stockholm, Sweden) was reconstituted in TRIS buffer $(\mathrm{pH}$ 9) according to the manufacturer's specifications.

Endotoxin standard derived from Escherichia coli 055 (Associates of Cape Cod, Woods Hole, Massachusetts, USA) was reconstituted with $5 \mathrm{ml} \mathrm{DW}$ to give a solution containing 100 $\mathrm{ng} / \mathrm{ml}$.

\section{ENDOTOXIN ASSAY}

Endotoxin $(0 \cdot 1 \mathrm{ml})$ prepared at various concentrations in DW was combined with $0.9 \mathrm{ml}$ of each test solution, usually treated plasma (described below) and DW. The prepared endotoxin solutions were vortex mixed and 75 $\mu \mathrm{l}$ was combined with $50 \mu \mathrm{l}$ of LAL at $4^{\circ} \mathrm{C}$ in a sterile microtitre plate (Disposable Products, North Carlton, Victoria, Australia). For the test solutions with the chromogenic substrate, $100 \mu \mathrm{l}$ of chromogen in buffer was also added to the wells. The fully loaded microtitre plate was placed at $37^{\circ} \mathrm{C}$ and the optical density at $405 \mathrm{~nm}$ (optical density 405) in each well was read using a Multiscan ELISA plate reader (Flow Laboratories Australasia). Optical density 405 readings were obtained starting from the end of the lag phase at $9 \cdot 5$, and then at $11,12 \cdot 5,14,16$, $18,20,22 \cdot 5,25,27 \cdot 5,30,33,36,40,44,49,54$, 60 and 70 minutes of incubation at $37^{\circ} \mathrm{C}$.

\section{KINETIC ANALYSIS OF OPTICAL DENSITY 405 READINGS}

Each optical density 405 reading was transformed to a percentage (optical density $405 \%$ ), taking the optical density 405 at 9.5 minutes (initial optical density) and the optical density 405 at 70 minutes (final optical density) for each curve as representing $0 \%$ and $100 \%$, respectively. Two slopes were then derived-a maximal slope and a logit slope. The maximal slope was the largest gradient between two adjacent time points on the optical density $405 \%$ curve. Logit transformation of optical density $405 \%$ was made using the formula:

logit optical density $405=\log (x /[100-x])$, where $x=$ optical density $405 \%$, using values of optical density $405 \%$ between 1 and $99 \%$. The logit slope was derived from the slope of the line of best fit derived by linear regression of logit optical density 405 against time in minutes.

Mathematical transformations and calculation of regression equations were performed using the Minitab statistical package.

\section{TREATED PLASMA SOLUTIONS}

Venous blood $(10 \mathrm{ml})$ was obtained from a volunteer who had no history of gastrointestinal or hepatic disease and whose high density lipoprotein cholesterol concentration was 1.63 $\mathrm{mmol} / \mathrm{l}$ by direct venepuncture into a sterile syringe. The blood was transferred to a sterile plastic container which contained 200 IU of heparin (Weddel Pharmaceuticals, Sydney, Australia). Separation of the plasma was achieved by centrifugation at $1000 \times g$ for 10 minutes at $4^{\circ} \mathrm{C}$. For serum, the blood was collected without heparin and allowed to stand until clotting was completed.

Plasma (5 ml) was added to $45 \mathrm{ml}$ of DW to give a $10 \%$ solution of plasma which was heated at $70^{\circ} \mathrm{C}$ for 20 minutes and then allowed to cool to $4^{\circ} \mathrm{C}$. The DW test solutions were heated in parallel with the diluted plasma. Mouse blood was obtained by direct cardiac puncture from the following inbred strains; $\mathrm{C} 3 \mathrm{H} / \mathrm{HeJ}$; C57BL/6; CBA; BALB/c; C57BL/ 10; and $\mathrm{C} 3 \mathrm{H} / \mathrm{HeN}$ (Walter and Eliza Hall Institute, Melbourne). The heat treatment of the plasma solutions, where applicable, always preceded the addition of endotoxin.

All data are presented as mean (SD). The coefficient of variation (CV) is calculated using the formula.

$$
\mathrm{CV}=(\mathrm{SD} / \text { mean }) \times 100 .
$$

\section{Results}

EFFECT OF TREATED PLASMA AND CHROMOGENIC SUBSTRATE

Representative progress curves for the optical density 405 over the time course of the reaction of LAL with endotoxin at a range of concentrations in four test solutions, and the corresponding logit optical density 405 curves, from an experiment performed in duplicate, are shown in fig 1 . The four test solutions were distilled water or heat treated $10 \%$ plasma, alone and in the presence of chromogenic substrate $(D$ and $\mathrm{D}+\mathrm{C}, \mathrm{P}$ and $\mathrm{P}+\mathrm{C}$, respectively). Whilst the optical density 405 (and optical density $405 \%$ ) follow a sigmoid curve, the logit transformation usually produces a linear relation between logit optical density 405 and time, with typical correlation coefficients $(r)$ of $>0.98$.

The gradients of the curves derived from fig 1 for the different concentrations of endotoxin in the four different types of test solution are presented in fig 2 as a double log plot. Note that for inclusion on the log scale, the endotoxin blank solution was arbitrarily assigned the concentration of $0.01 \mathrm{ng} / \mathrm{ml}$, which is the sensitivity limit of the LAL reagent, as labelled by the manufacturers.

The course of the reaction at different concentrations of endotoxin depended on the type of test solution studied. In the absence of chromogen - that is, when D is compared with $P$ - the effect of heat inactivated plasma on the LAL reaction is two-fold; it increases the optical density 405 increment-that is, from initial to final optical density 405 (fig 1)-and it increases the rate at which the reaction proceeds for all concentrations of endotoxin (fig 2).

The addition of chromogen produces a large increase in optical density 405 which obscures the difference in optical density 405 due to plasma-that is, $D+C$ compared with $P+C$ 
Figure 1 Progress of LAL reaction as optical density $405(\times 1000)$ or as logit optical density 405 at different concentrations of endotoxin in the presence of two test solutions

$(D=$ distilled water $P=10 \%$ plasma) alone or in the presence of

chromogenic substrate

$(+C)$.
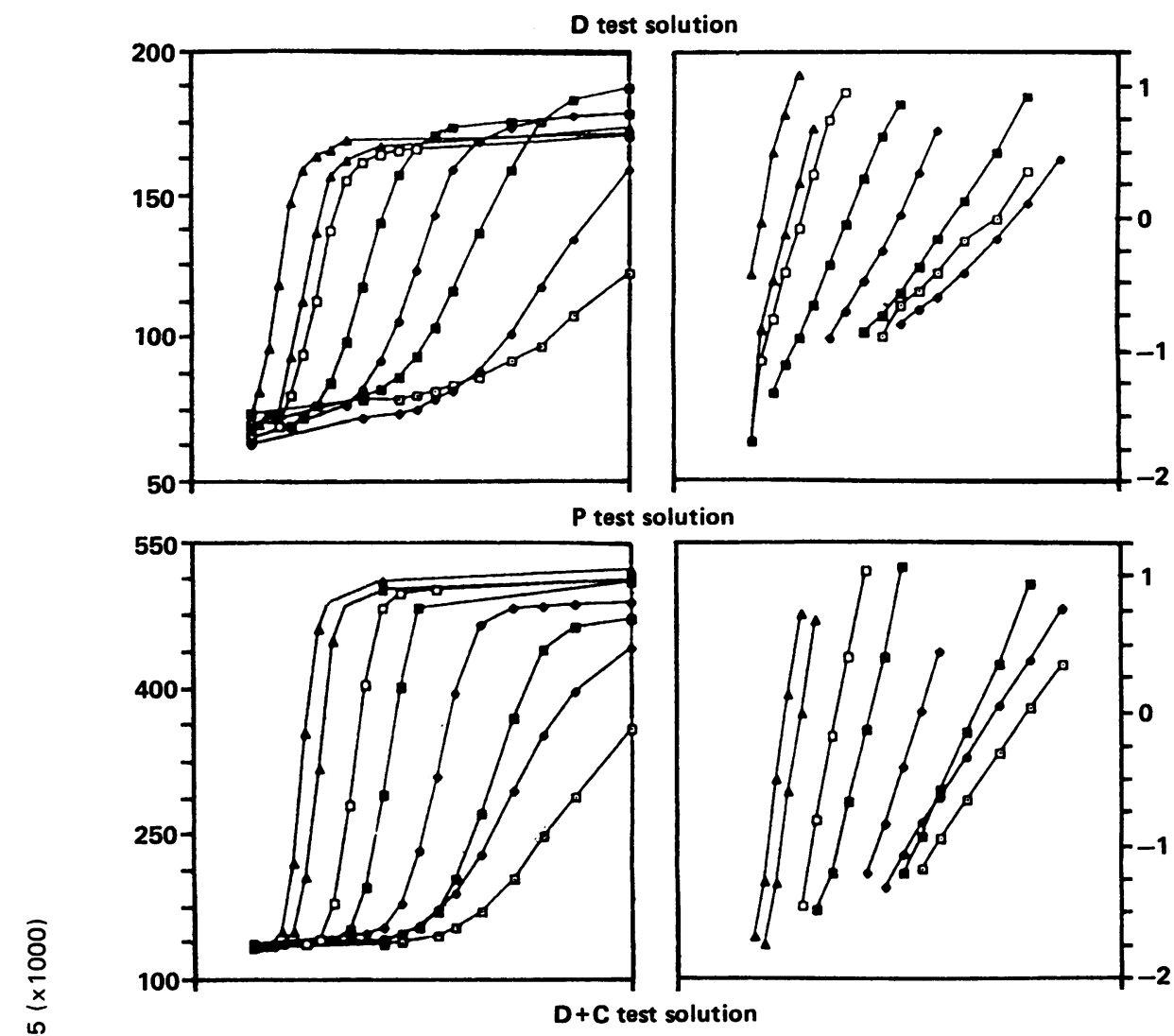

ஓ̊
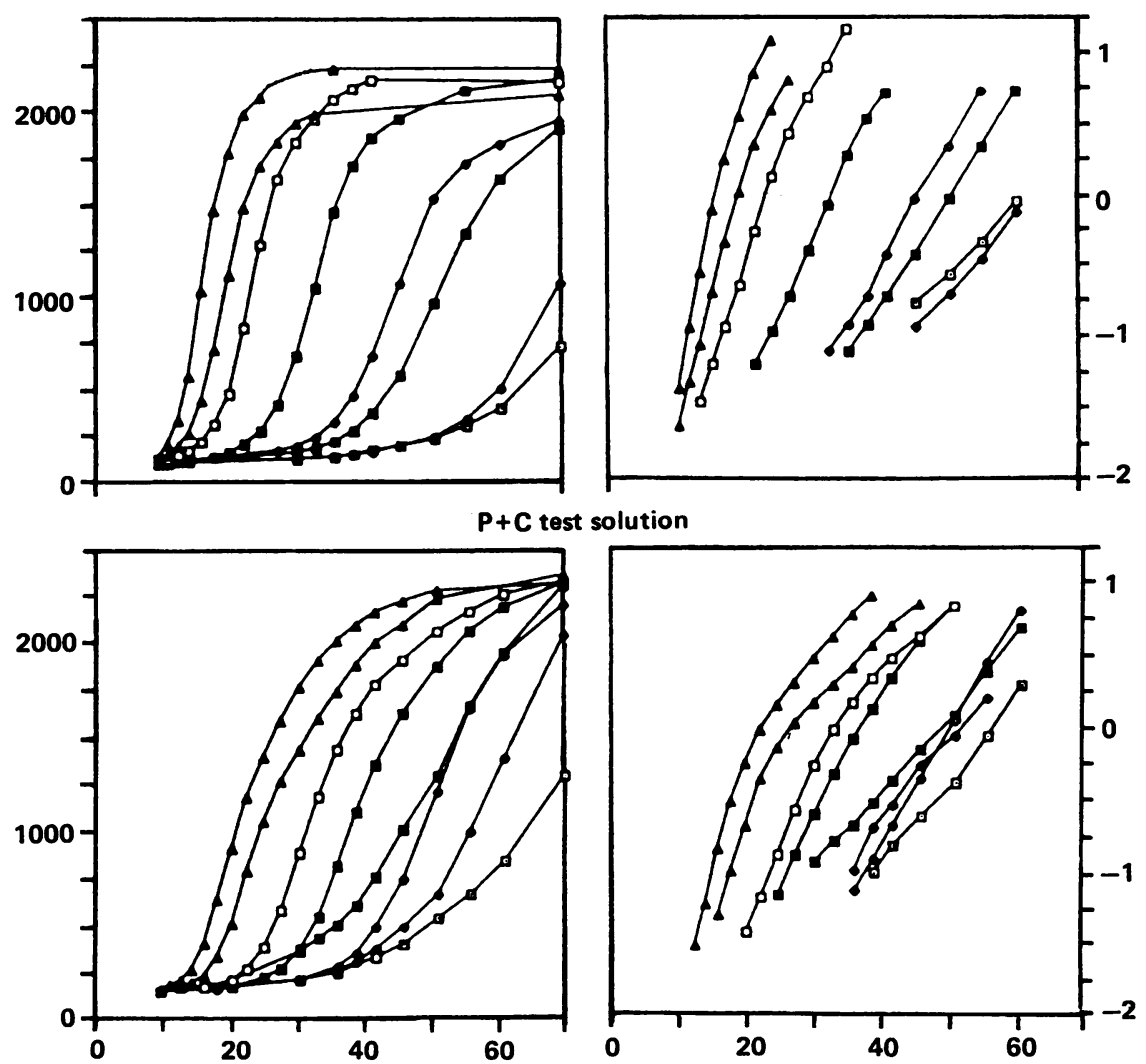

Time (minutes)

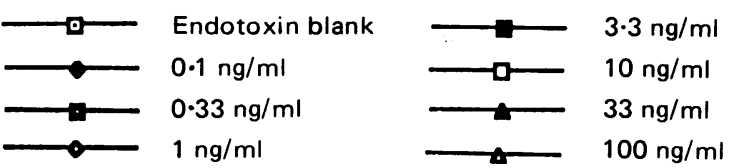

Test solutions:

$D=$ distilled water alone

$P=10 \%$ plasma alone

$D+C=$ distilled water and chromogenic substrate

$P+C=10 \%$ plasma and chromogenic substrate 
Figure 2 Kinetic standard dose-response curves for endotoxin as a log plot showing the effects of test solution and chromogenic substrate. The response is either the maximal slope of the percentage transformed optical density 405 curves or the slope of the line of best fit of the logit optical density 405 curves shown in fig 1 . The endotoxin blank solution was assigned the concentration of 0.01 $\mathrm{ng} / \mathrm{ml}$, as this was the sensitivity limit of the $L A L$ reagent as labelled by the manufacturer.

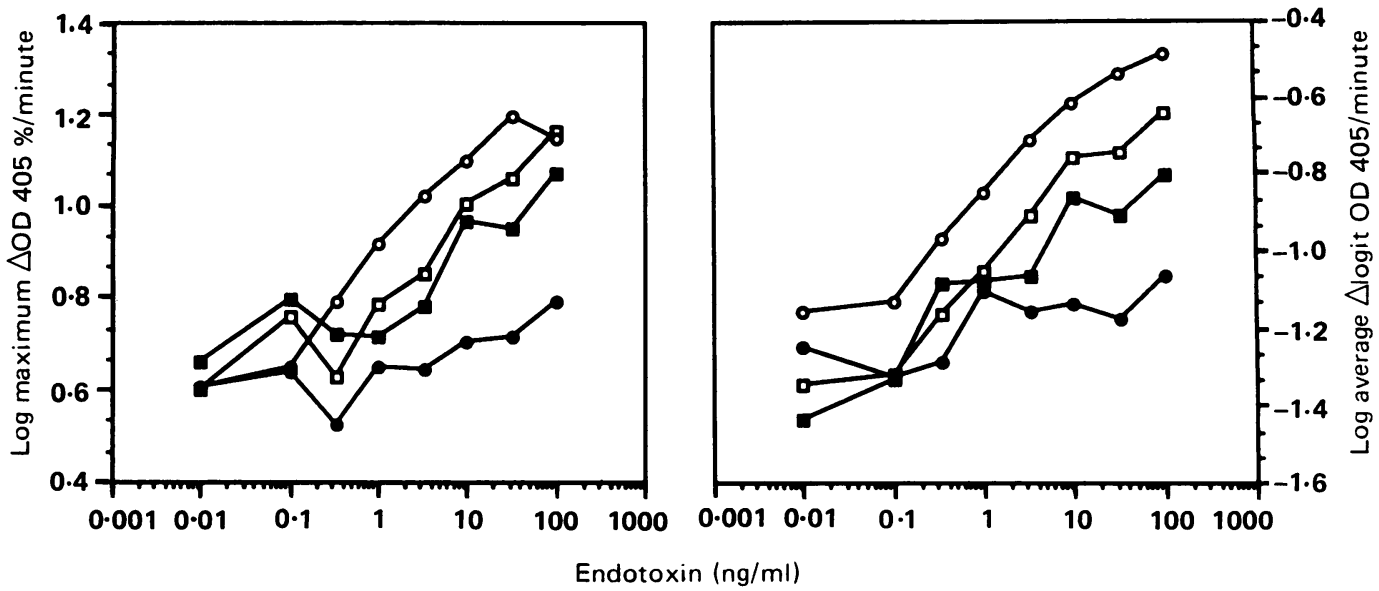

\begin{tabular}{|lll|}
\hline - & Distilled water alone & - Distilled water with chromogenic substrate \\
- $10 \%$ plasma alone & - $10 \%$ plasma with chromogenic substrate \\
\hline
\end{tabular}

(fig 1). In the presence of chromogen and heat inactivated $10 \%$ plasma there was a considerable delay in reaching the maximum optical density value. Consequently, the rate at which the reaction proceeds, expressed either as a logit or as a percentage increase in optical density 405 , is decreased in the $P+C$ solution at all concentrations of endotoxin except those below $0.33 \mathrm{ng} / \mathrm{ml}$, compared with the test solution without chromogen (fig 2 ).

EFFECT OF VARIOUS TREATED PLASMA AND SERUM SOLUTIONS

The time course of the LAL reaction in the presence of different treated plasma solutions was observed at eight concentrations of endotoxin over the range 0.033 to $100 \mathrm{ng} / \mathrm{ml}$ and endotoxin blank. The results of the experiments are presented as the relative effects of the treated plasma solutions on both the absolute increase in optical density 405 and the rate of the LAL reaction. The effect of treated plasma solutions on the absolute increase in optical density 405 was determined from wells containing the four highest concentrations of endotoxin $(100,33,10$ and $3.3 \mathrm{ng} / \mathrm{ml})$, for which the final optical density 405 readings were always representative of the upper plateau of the respective sigmoid curves. The mean and standard deviation of the initial optical density 405 , final optical density 405 , and the difference

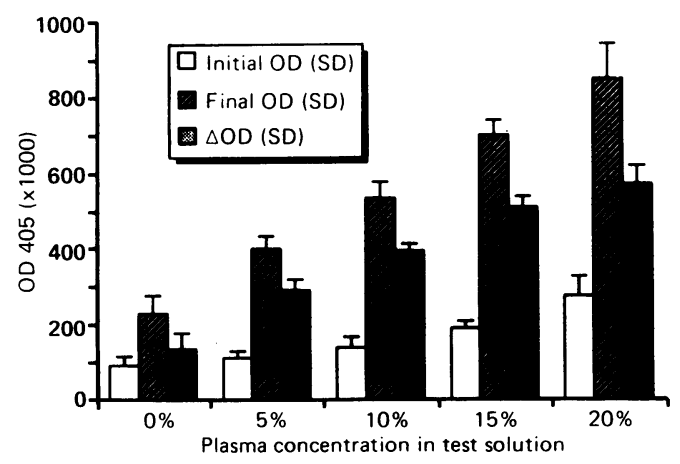

Figure 3 Optical density data for solutions with distilled water or different concentrations of dilution and heat treated plasma at set concentrations of endotoxin. between initial and final optical density 405 were obtained using the readings from these wells. The effect of the treated plasma solutions on the rate of the LAL reaction was determined from the logit slopes which are presented in a double log plot against the full range of concentrations of endotoxin.

Although the absolute change in optical density was proportional to the concentration of plasma present (fig 3), the rate of change of the logit optical density 405 curves at any given concentration of endotoxin was similar in the presence of the different concentrations of treated plasma (fig 4); the corresponding rates in the distilled water test solution were decreased by comparison.

The absolute change in optical density was similar in solutions containing $10 \%$ plasma (387 (19.9)) and 10\% serum (379 (12.3)) compared with the DW solution (90 (12.4)). The rate of change was similar in the $10 \%$ plasma solutions and the $10 \%$ serum solution at all concentrations of endotoxin (fig 5).

The importance of heat treatment of the plasma solution in the interaction with Limulus reagent and endotoxin was tested by comparing the progress of optical density

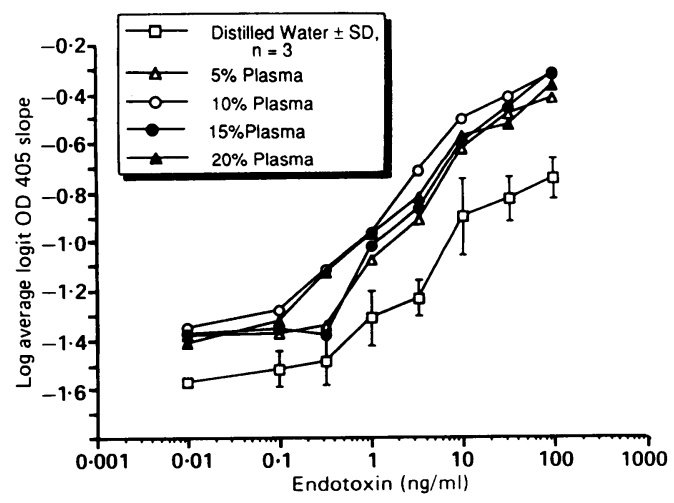

Figure 4 Dose-response curves for endotoxin in solutions containing different concentrations of plasma. The response is the slope of the line of best fit of the logit optical density 405 plot. For inclusion on the log scale, the density 405 plot. For inclusion on the log scale, the
endotoxin blank solution was arbitrarily assigned the concentration of $0.01 \mathrm{ng} / \mathrm{ml}$, which is the sensitivity limit of the $L A L$ reagent as labelled by the manufacturers. 
Figure 5 Dose-response curves for endotoxin in treated solutions of either serum, plasma, or distilled water alone. The response is the slope of the line of best fit of the logit optical inclusion on the log scale, the endotoxin blank solution was arbitrarily assigned the concentration of $0.01 \mathrm{ng} / \mathrm{ml}$, which is the sensitivity limit of the $L A L$ reagent as labelled by the manufacturers. density 405 plot. For

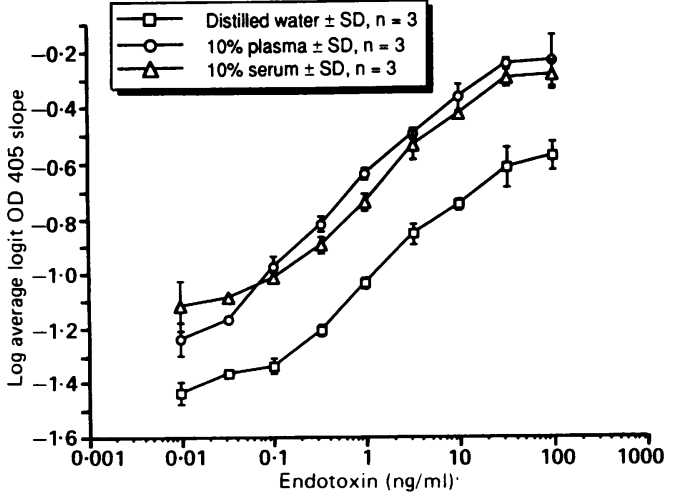

development in solutions of heat treated $10 \%$ plasma with that in plasma solutions not exposed to heat. Three differences were observed when the plasma solution was not exposed to heat treatment. The absolute change in optical density (349 (11.3) with heat treated plasma $v 96(49.6)$ with non-heat treated plasma) was reduced, the rate of change in optical density for any given concentration of endotoxin was reduced, and the lag phase which preceded the increase in optical density was extended (data not shown).

With $10 \%$ solutions of mouse plasma, the absolute change in optical density was marginally higher (384 (30.9)) than for a $10 \%$ solution of human plasma (300 (7.5)), compared with the DW solution $(90(8 \cdot 7))$. With all the mouse plasma solutions, the rate of change at any given concentration of endotoxin was intermediate between those with the DW solution and the solution of human plasma. There was no difference between plasma from strains of mice unresponsive to endotoxin $(\mathrm{C} 3 \mathrm{H} / \mathrm{HeJ})$ and plasma from strains responsive to endotoxin (fig 6).

\section{ASSAY VARIABILITY}

The intra-assay and interassay coefficients of variation were calculated for logit based kinetic assays using DW solutions, as well as for assays using heat treated $10 \%$ plasma solutions. The intra-assay $\mathrm{CV}$, estimated by determination of lipopolysaccharide content in 44 replicates of a solution containing $100 \mathrm{ng} / \mathrm{ml}$ endotoxin, was $23.1 \%$ with the DW solution, and $17.3 \%$ with the treated plasma solution. The intra-assay

Figure 6 Dose-response curves for endotoxin in treated solutions of either distilled water alone or $10 \%$ solutions of human or inbred mouse plasma. The response is the slope of the line of best fit of the logit optical density 405 plot. For inclusion on the log scale, the endotoxin blank
solution was arbitrarily assigned the concentration of $0.01 \mathrm{ng} / \mathrm{ml}$, which is the sensitivity limit of the $L A L$ reagent as labelled by the manufacturers.

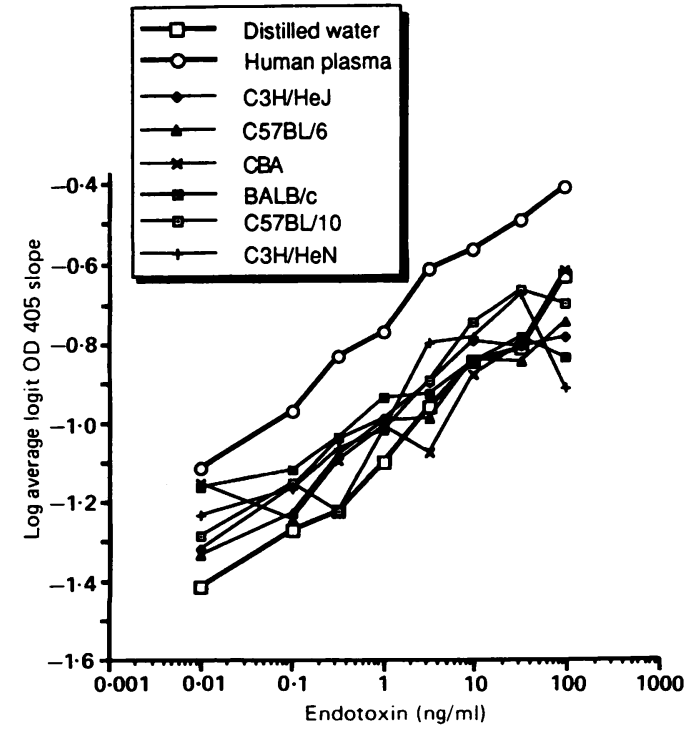

$\mathrm{CV}$ for the measurement of endotoxin at a dose of $100 \mathrm{ng} / \mathrm{ml}$ added to treated plasma samples of eight normal volunteers was $60 \%$. The interassay $C V$, estimated by measuring the lipopolysaccharide content in the same solution containing $33 \mathrm{ng} / \mathrm{ml}$ of endotoxin in five assays performed on different days, was $64.6 \%$ with the distilled water and $56.0 \%$ with the treated plasma test solutions.

\section{Discussion}

In the endotoxin activated LAL reaction, while the absolute change in optical density is determined by the concentration of clottable protein or substrate, it is the rate of change in optical density which is proportional to the concentration of endotoxin present. ${ }^{3}$ We examined the effect of various preparations of plasma and serum on both aspects of the progress of turbidity development in the LAL reaction. The monitoring of the changing optical density was facilitated by interfacing the optical density reader with a microcomputer.

The methodology used in this study differs in three respects from that of earlier studies: the plasma was diluted and heat treated before endotoxin was added and such heat treated plasma was not inhibitory to the LAL reaction. Secondly, in order to meet the criteria of a "truly kinetic" rather than a "pseudo kinetic" methodology, the monitored period must correspond to the period of LAL activation. ${ }^{14}$ To study the effect of the chromogenic substrate, therefore, we combined the reagents in a single step incubation. Thirdly, we compared two methods of kinetic analysis, one using logit transformed optical density data. ${ }^{15}$ The logit transformation is commonly used as a means of obtaining a straight line relation from sigmoidal curves such as those seen with bioassay data.

In this study kinetic analysis using slopes from either percentage or logit transformed optical density 405 data gave similar doseresponse correlations. The logit slope method has an advantage of being calculated using more than two data points for each reaction curve.

In the absence of chromogen dilution and heat treated plasma increased the total increase in optical density 405 (fig 1 ) and also accelerated the rate of increase in optical density 405, standardised as either optical density $405 \%$ or logit optical density 405 (fig 2). With chromogenic substrate present, however, the contribution of plasma to the total increase in optical density 405 was obscured by the development of chromogen. Moreover, there was a considerable delay in reaching the maximum optical density value which is reflected in the lower rates of increase in optical density 405 at concentrations of endotoxin above $0.33 \mathrm{ng} /$ $\mathrm{ml}$.

Nitsche et $a l^{6}$ reported that heat treated fibrinogen reacts with coagulogen to form an adduct to produce an increase in turbidity readings; adduct formation occurs rapidly ( $75 \%$ complete within 60 seconds), does not require the presence of activated LAL proenzyme, and is more apparent with turbidimetric than chromogenic $\mathrm{LAL}$ assay techniques using end point methodology. The authors claim that in the turbimetric assay the 
concentration of fibrinogen should be measured and the end point readings adjusted to allow for this effect. Our observations on the effect of plasma on initial optical density 405 readings are similar to those of Nitsche et al. ${ }^{6}$ Moreover, the effect is even more apparent at the completion of the LAL reaction (fig 3), suggesting that the adduct formed with cleaved coagulogen is more optically dense than that formed with native coagulogen.

The effect of treated plasma on the kinetics of the LAL reaction may be due to the amplification effect from high density lipoprotein, permitting a more efficient interaction between endotoxin and LAL. ${ }^{7}$ The rate of increase at any given concentration of endotoxin was uniform at the various concentrations of plasma tested (fig 4), suggesting that the amount of high density lipoprotein present was not limiting at any of the concentrations of plasma and endotoxin examined using plasma from this single donor.

The effect of plasma from the $\mathrm{C} 3 \mathrm{H} / \mathrm{HeJ}$ mouse strain, a strain that is unresponsive to endotoxin, was no different to the effect of plasma from endotoxin responsive strains (fig 6 ). This finding contrasts with a report that a heat stable plasma factor, which assists the interaction between the third component of complement and antigen-antibody complexes, is deficient in this strain. ${ }^{16}$

In contrast to previous reports, we found that the LAL reactivity with endotoxin was similar in the presence of serum and plasma (fig 5). In our study the temperature sensitive esterases known to be present in plasma and serum were heat inactivated before the addition of endotoxin. The results of the studies with non-heat treated plasma are consistent with some inactivation of endotoxin in this plasma, a process which is known to be time dependent. ${ }^{17} 18$ The similar behaviour of dilution and heat treated serum and plasma suggests that the greater inhibitory effect of serum observed by others ${ }^{1319}$ is due to the presence of a factor in serum which is sensitive to heat inactivation.

The intra-assay $\mathrm{CV}$ with the simultaneous assay of endotoxin in plasma from different subjects greatly exceeds that observed when pooled plasma is used. ${ }^{13}$ We have found that this also applies when comparing treated plasma samples of different subjects, although to a lesser extent.

The interassay variability observed here using kinetic methodology was $56.0 \%$ with the treated $10 \%$ plasma solution. This is higher than that reported for end point methodology where the interassay variability is $25 \%{ }^{7} \mathrm{Kin}$ etic methodology, however, has the advantage of covering a four log ten-fold range of endotoxin concentrations in a single step assay, an important attribute given the greater than three log ten-fold variability of endotoxin in the plasma of patients presenting with Gram negative bacterial sepsis. ${ }^{20}$ The assay variability using kinetic methodology is comparable with that reported for the cumbersome serial dilution gel clot methodology, where the accuracy is within plus or minus 1 two-fold dilution step. ${ }^{2122}$ With kinetic methodology, however, this is achieved without the need for serial dilutions and hence with greater economy of
LAL reagent, especially with the use of microtitre volumes.

It should be appreciated that any effects of plasma on LAL kinetics will not be recognised in end point readings. More importantly, however, we found that the kinetics of the LAL reaction are affected by the copresence of chromugenic substrate and treated plasma. For this reason, the turbidimetric assay would be preferable to the chromogenic assay when kinetic methodology is used for the quantitative assay of endotoxin in plasma. Moreover, the chromogen-like effect of treated plasma raises the possibility of its routine use as a reagent to improve the assay for the quantitation of endotoxin in non-plasma samples such as urine (Hurley, Louis and Tosolini, unpublished observations).

JC Hurley was supported by a medical postgraduate scholarship from the National Health and Medical Research Council.

1 Levin J, Bang FB. The role of endotoxin in the extracellular coagulation of Limulus blood. Bull Johns Hopkins Hosp 1964;115:265-74.

2 Elin RJ, Hosseini J. Clinical utility of the Limulus amoebocyte lysate (LAL) test. Prog Clin Biol Res 1985; 189:307-27.

3 Levin J, Bang FB. Clottable protein in Limulus: Its localization and kinetics of its coagulation by endotoxin. Thromb Diath Haem 1968;19:186-97.

4 Johnson KJ, Ward PA, Goralnick S, Osborn MJ. Isolation from human serum of an inactivator of bacterial lipopolysaccharide. Am J Pathol 1977;88:559-74.

5 Levin J, Tomasulo PA, Oser RS. Detection of endotoxin in human blood and demonstration of an inhibitor. J Lab Clin Med 1970;75:903-11.

6 Nitsche D, Kriewitz M, Seifert J, Hamelmann H. Investigations on an essential plasma factor disturbing the photometric determination of endotoxin in plasma samples with the Limulus test. Prog Clin Biol Res 1987;231:331-40.

7 Harris RI, Stone PCW, Stuart J. An improved chromogenic substrate endotoxin assay for clinical use. J Clin Pathol substrate endotoxin

8 Nandan R, Brown DR. An improved in vitro pyrogen test: to detect picograms of endotoxin contamination in intravenous fluids using Limulus amoebocyte lysate. J Lab Clin Med 1977;89:910-8.

9 Zhang GH, Baek L, Koch C. New microassay for quantitation of endotoxin using Limulus amoebocyte lysate combined with enzyme-linked immunosorbent assay. J Clin Microbiol 1988;26:1464-70.

10 Iwanaga S, Morita T, Harada $\mathrm{T}$, et al. Chromogenic substrates for horseshoe crab clotting enzyme: Its application for the assay of bacterial endotoxins. Haemostasis 1978;7:183-8.

11 DuBose DA, Lemaire $M$, Basamania K, Rowlands J. Comparison of plasma extraction techniques in preparation of samples for endotoxin testing by the Limulus tion of samples for endotoxin testing by the Limulus

12 Ditter B, Becker KP, Urbaschek R, Urbaschek B. Detection of endotoxin in blood and other specimens by evaluation of photometrically registered LAL-reaction-kinetics in microtiter plates. Prog Clin Biol Res 1982;93:385-92.

3 Cohen J, McConnell JS. Observations on the measurement and evaluation of endotoxemia by a quantitative Limulus lysate microassay. J Infect Dis 1984;150:916-24.

14 Novitsky TJ. Development of a quantitative LAL assay. In: Novitsky TJ, Ed. LAL update. Vol 3. Woods Hole, Massachusetts: Association of Cape Cod, 1985.

15 Hewlett PS, Plackett RL. The interpretation of quantal responses in biology. London: Edward Arnold, 1979.

16 Hoffmann MK. Serum from LPS nonresponder C $3 \mathrm{H} / \mathrm{HeJ}$ mice does not support the formation of functional EAC mice does not support the formation

17 Obayashi KT, Tamura H, Tanaka S, Ohki M, Takahashi S, Kawai $T$. Endotoxin inactivating activity in normal and Kawai T. Endotoxin inactivating activity in normal and pathological

18 Olofsson P, Olofsson C, Nylander G, Olsson P. Endotoxin inactivation in plasma from septic patients: an in vitro study. World J Surg 1986;10:318-23.

19 Novitsky TJ, Roslansky PF, Siber GR, Warren HS. Turbidimetric method for quantifying serum inhibition of Limulus amoebocyte lysate. J Clin Microbiol 1985; 20:211-6.

20 Shenep JL, Flynn PM, Barrett FF, Stidham GL, Westenkirchner DF. Serial quantitation of endotoxaemia and bacteraemia during therapy for Gram negative bacterial sepsis. J Infect Dis 1988;157:565-8.

21 Gardi A, Arpagaus GR. Improved microtechnique for endotoxin assay by the Limulus amoebocyte lysate test. Analyt Biochem 1980;109:382-5.

22 Shenep JL, Mogan KA. Kinetics of endotoxin release during antibiotic therapy for experimental $\mathrm{Gram}$-negative bacterial sepsis. J Infect Dis 1984;150:380-8. 